\title{
Optical Design and Predicted Performance of the WFIRST Phase-B Imaging Optics Assembly and Wide Field Instrument
}

\author{
Bert A Pasquale ${ }^{\mathrm{a}}$, Thomas Casey ${ }^{\mathrm{b}}$, Catherine Marx ${ }^{\mathrm{a}}$, Guangjun Gao ${ }^{\mathrm{c}}$, Nerses Armani ${ }^{\mathrm{d}}$, David \\ Content $^{\mathrm{a}}$, John Hagopian ${ }^{\mathrm{e}}$, Alden Jurling ${ }^{\mathrm{a}}$, Clifton Jackson ${ }^{\mathrm{d}}$, Alice Liu ${ }^{\mathrm{a}}$, Art Whipple ${ }^{\mathrm{f}}$, Jacob Murray ${ }^{\mathrm{g}}$ \\ aNASA/Goddard Space Flight Center, Greenbelt, MD 20771, bAS\&D, Inc. 7000 Muirkirk Meadows \\ Dr., Beltsville, Maryland 20705, 'Sigma Space Inc. 4600 Forbes Blvd, Lanham-Seabrook, MD \\ 20706, dSGT Inc. 7701 Greenbelt Road, Greenbelt, MD 20770, ${ }^{\mathrm{e} A T A}$ Aerospace Inc., Greenbelt MD \\ 20770ATA Aerospace Inc, fConceptual Analytics Inc., Glenn Dale, MD 20769, ${ }^{\text {g }}$ Rose-Hulman \\ Institute of Technology, Terre Haute, IN 47803 \\ Bert.Pasquale@nasa.gov, Thomas.Casey@nasa.gov
}

\begin{abstract}
Now in Phase-B, the architecture of the Wide-Field Infra-Red Survey Telescope (WFIRST) payload has matured since 2013 to accommodate various opto-mechanical constraints. Based on a 2.4-meter aperture Forward Optical Assembly (FOA), the Imaging Optics Assembly (IOA) provides corrected optical fields to each on-board instrument. Using a Three Mirror Anastigmat (TMA) optical design, the Wide-Field Channel (WFC) provides $\sim 1 / 3$-square degree of instantaneous field coverage at 0.11 arcsecond pixel scale. The WFC as-built predictive analysis anticipates near diffraction-limited imaging over a focal plane of 300.8 million pixels, operating in seven panchromatic bands between $0.48-2.0 \mu \mathrm{m}$, or a 1-octive multi-spectral imaging mode from $\sim 0.95-1.93 \mu \mathrm{m}$. The IOA provides the Coronagraph Instrument (CGI) a collimated beam with very specific wavefront constraints. We present configuration changes since 2013 that improved interfaces, improved testability, and reduced technical risk. We provide an overview of our Integrated Modeling results, performed at an unprecedented level for a phase-A study, to illustrate performance margins with respect to static wavefront error, jitter, and thermal drift.
\end{abstract}

Keywords: Space Instrumentation, Astronomical Optics, Imaging Systems, Telescopes, Dark Energy, Spectroscopy, Systems Engineering, Integrated Modeling

\section{INTRODUCTION AND CURRENT STATUS}

NASA's Wide Field Infrared Survey Telescope (WFIRST) ${ }^{[1]}$ is being designed to deliver unprecedented capability in dark energy and exoplanet science, and to host a technology demonstration coronagraph for exoplanet imaging and spectroscopy. Scheduled for launch in 2025, WFIRST will fly in a Sun-Earth L2 orbit with a primary mission life of 5 years plus a Guest Observer program. The instruments and the spacecraft are designed to be robotically flight serviceable. The observatory design has matured since the initial Pre-Phase-A designs ${ }^{[2,3,4]}$; here we present a comprehensive description of the observatory configuration as refined during the WFIRST Phase- $\mathrm{A}^{[5,6]}$ design cycle and now into Phase-B. This paper documents these changes and will emphasize the optical system and its performance in the systematic context.

WFIRST relies on the reuse of an existing $2.4 \mathrm{~m}$ aperture Forward Optics Assembly (FOA) donated to NASA by another agency in 2012, consisting of the Primary Mirror (PM) and Secondary Mirror (SM) along with their supporting structure. These are now the first two mirrors of the Three-Mirror Anastigmat (TMA) optical form of the Wide Field Channel (WFC). With only minor modifications to this existing FOA hardware, WFIRST will enable wide area multi-band NIR surveys via imaging and spectroscopy to explore the expansion history of the universe, investigate the growth of large scale structures within the universe, and perform a microlensing survey to discover and catalog extra-solar planets within our galaxy ${ }^{[7]}$. The observatory Wide Field Instrument (WFI) boasts a 300 megapixel visible to near-infrared $(0.48$ to 2 microns) highly-sensitive 18-Sensor Chip Assembly (SCA) $\mathrm{HgCdTe}$ focal plane array with multiple imaging and spectrographic modes. This enables very rapid science surveys by imaging of a $\sim 0.28^{\circ}$ sq. field of view at .11 asec/pixel, which is approximately 90-220 times larger than the Hubble Space Telescope instruments. In addition, WFIRST will accommodate a technology demonstration of a starlight suppression coronagraph enabling direct imaging and 
spectroscopy of exoplanets and debris disks. A composite truss structure meters both instruments to the telescope assembly.

Recent program milestones include:

- WFIRST Science Investigation Teams (SITs) selected December 2015 (204 members)

- Completed Key Decision Point-A (KDP-A; the beginning of Phase-A) in February 2016

- Preliminary Phase-A Point Design (v 7.6.8) frozen September 25, 2016

- TRL-6 on WFI H4RG detectors achieved December 2016

- Coronagraph testbed achieved dynamic contrast of 10-08 in Jan 2017

- Completed of Wide Field Opto-Mechanical Assembly (WOMA) Phase-A studies in May 2017

- Completed Key Decision Point-B (KDP-B; the beginning of Phase-B) in May 2018

- Phase-B Baseline Optical Design (v 8.5.4) frozen June 6, 2018

- Beginning of engagement with WOMA vendor (Ball Aerospace) in June 2018

This paper will present an overview of the innovations first introduced in Phase-A and now refined in the Phase-B configuration, summarize key innovations resulting from our Phase-A study, and document integrated modeling results performed in support of these changes. The configuration modifications include:

- The implementation of an Instrument Carrier structure to meter the WFI and Coronagraph independently of the donated FOA structure.

- The relocation of the Tertiary Mirror from the WFI to the Optical Telescope Assembly.

- The change from active to passive cooling for the $\sim 100 \mathrm{~K}$ WFI focal place assembly.

- The implementation of the WFC Fold Mirror 1 and Focal Plane Assembly actuation mechanisms

- The loss of the Integral Field Channel Instrument from the payload

As will be seen, the combined efforts of the WFIRST team in Phase-A on these key trades ${ }^{[8]}$ have resulted in significant risk reduction for the WFIRST program to make the Phase-B design as robust and future-proof as possible.

\section{WFIRST OBSERVATORY AND PAYLOAD OVERVIEW}

The WFIRST Observatory consists of an Integrated Payload Assembly (IPA; see Figure 1) and a Spacecraft (S/C). As will be discussed below, the IPA consists of components of the Optical Telescope Assembly (OTA), an Instrument Carrier (IC), the Wide Field Instrument (WFI) and the Coronagraph Instrument (CGI). As discussed in previous publications ${ }^{[4,5]}$, the observatory's Imaging Optics Assembly (IOA) uses a repurposed $2.4 \mathrm{~m}$ Forward Optical Assembly (FOA) donated from another agency, repurposed by NASA for scientific use.

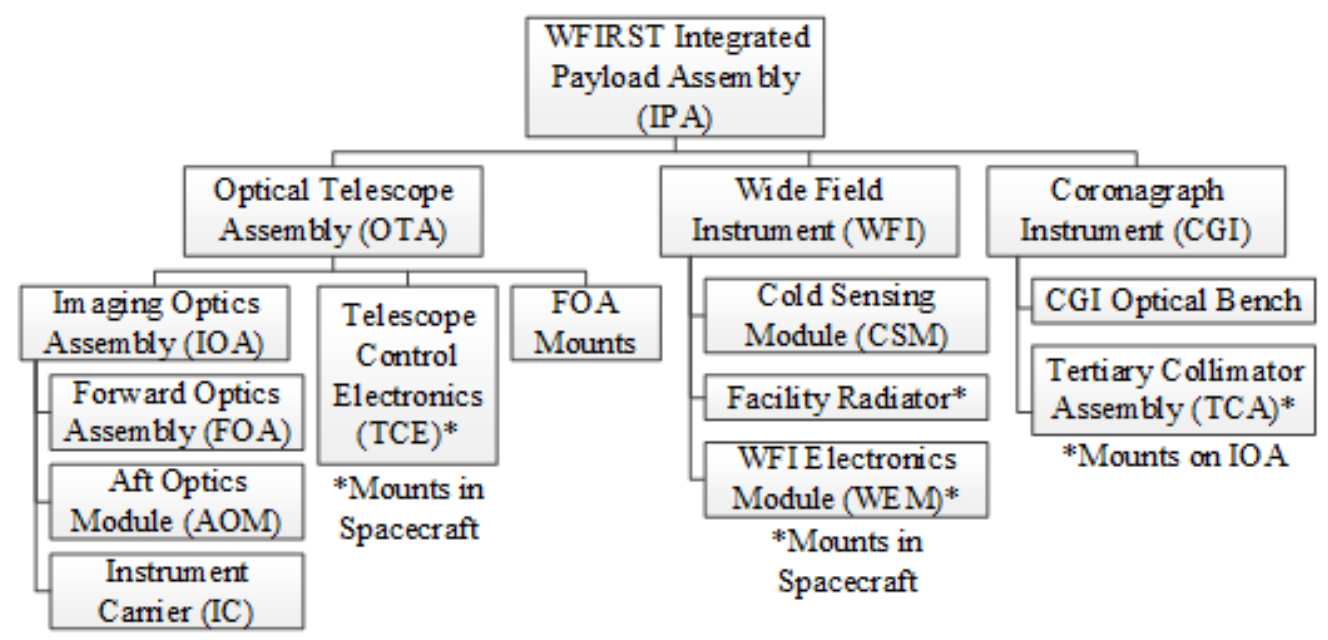

Figure 1. WFIRST Hardware Breakdown Structure

The full IPA sits atop the Spacecraft. The OTA consists of the donated FOA integrated with an Aft Optics Module (AOM) and supported by the Instrument Carrier (IC) via FOA mounts. The OTA is controlled by its Telescope Control 
Electronics (TCE) mounted within the WFIRST Spacecraft. The AOM/WFC design also accommodates the volume constraints of the CGI pickoff and Tertiary Collimator Assembly. The IC provides the rigid truss off the spacecraft, while maintaining the lines of action from the SC through to the FOA. This creates an ultra-stable structure for the entire payload. See Figure 2.
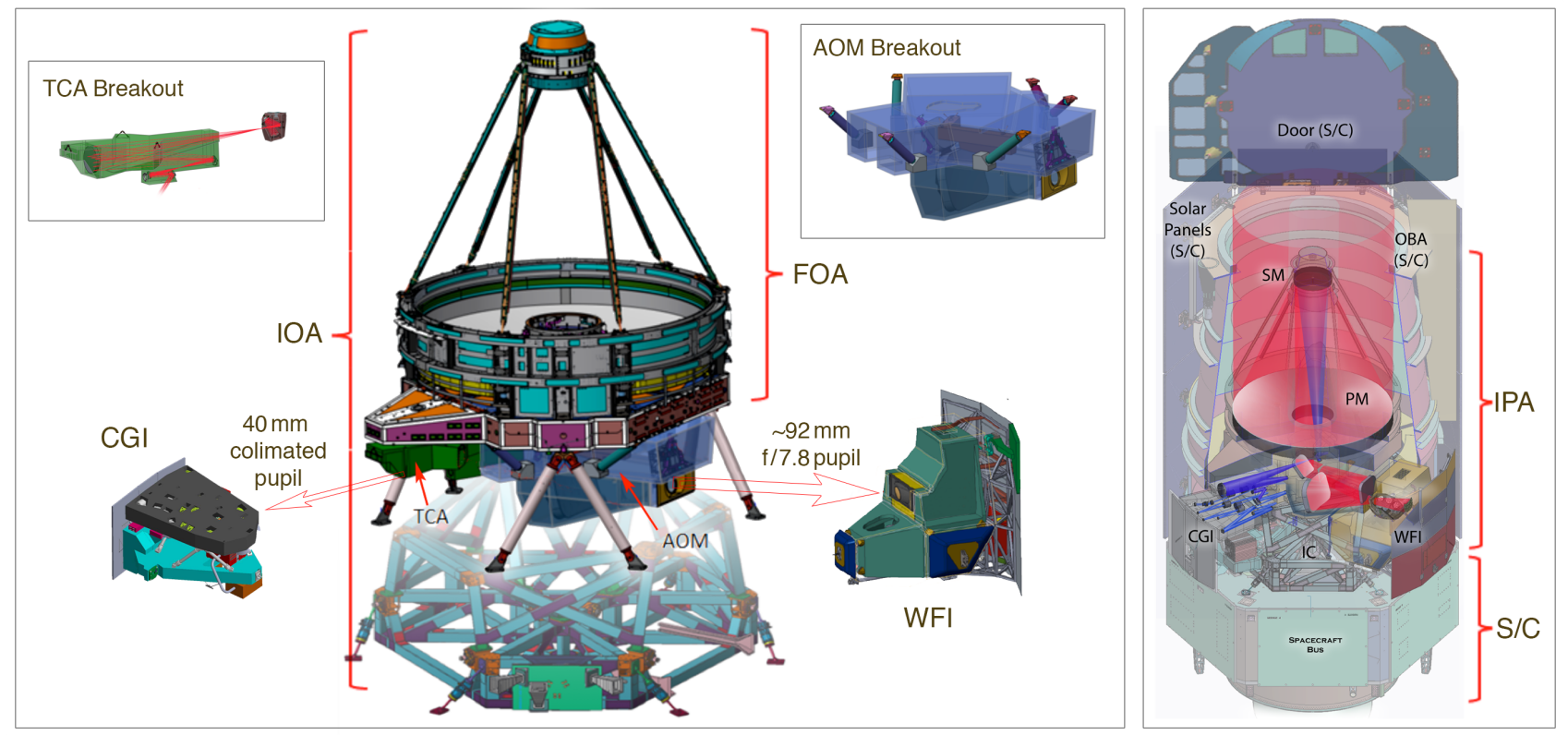

Figure 2. Some assembly required: The components of the WFIRST IOA are built around the heritage FOA, with the AOM and TCA directly attached. The WFC exit pupil of the IOA mates with the WFI, while the CGI receives a collimated pupil. The IOA and IC make up the IPA, which is supported on the S/C bus.

Functionally, the first two optics of the OTA's Three Mirror Anastigmat imaging system is in the common path for all WFIRST science modes. The existing FOA will undergo an optical prescription change for the WFIRST mission. (PM: R: 5671.1342, K: -0.9728630311 / SM: R: 1299.6164, K: -1.6338521231). Figure 3 shows the scope of the existing hardware.
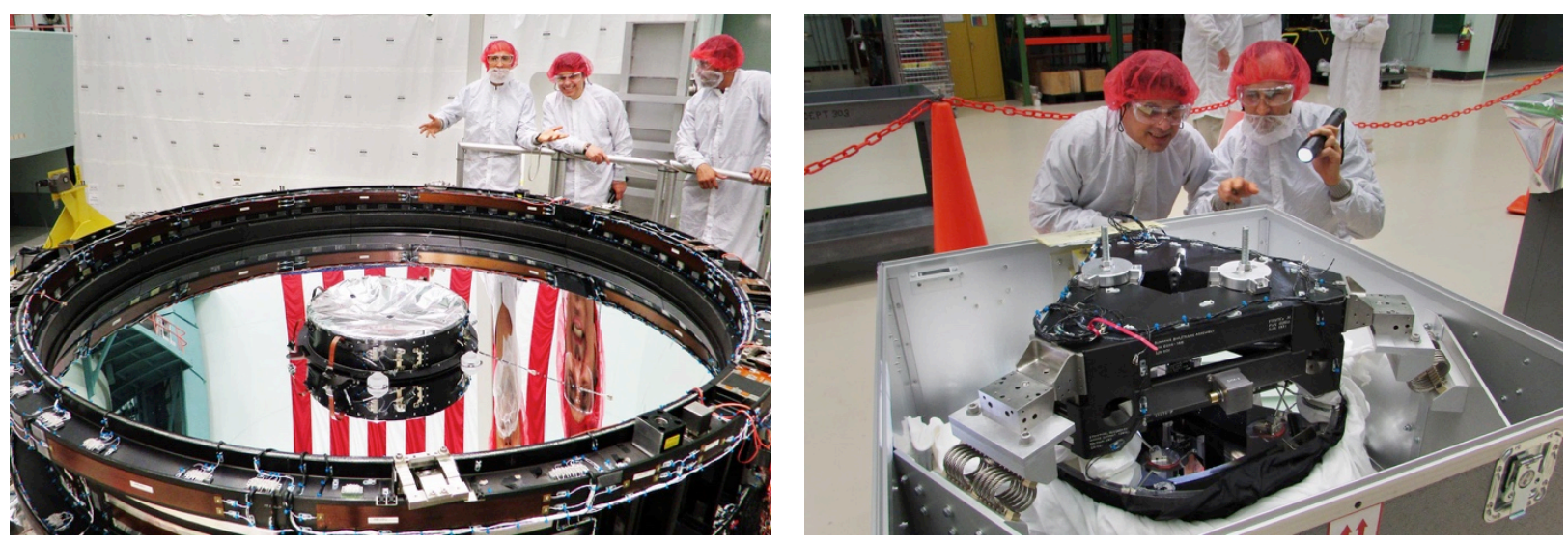

Figure 3. (a) The WFIRST Primary Mirror (PM) during a 2016 hardware inspection. (Left to right: Bert Pasquale; Optical Designer, Nerses Armani; Mechanical Designer, and Marcello Rodriguez; Payload System Engineer.) (b) N. Armani and B. Pasquale with the WFIRST Secondary Mirror Assembly. (The back of the mirror can be seen suspended under the assembly.) Images are ITAR-cleared.

The passively isolated FOA Mounts and the AOM are new hardware specifically designed for WFIRST. As a result of one of the trades described in this paper, the Tertiary Mirror of the TMA is now mounted in the AOM instead of the WFI as can be seen in Figure 4. 


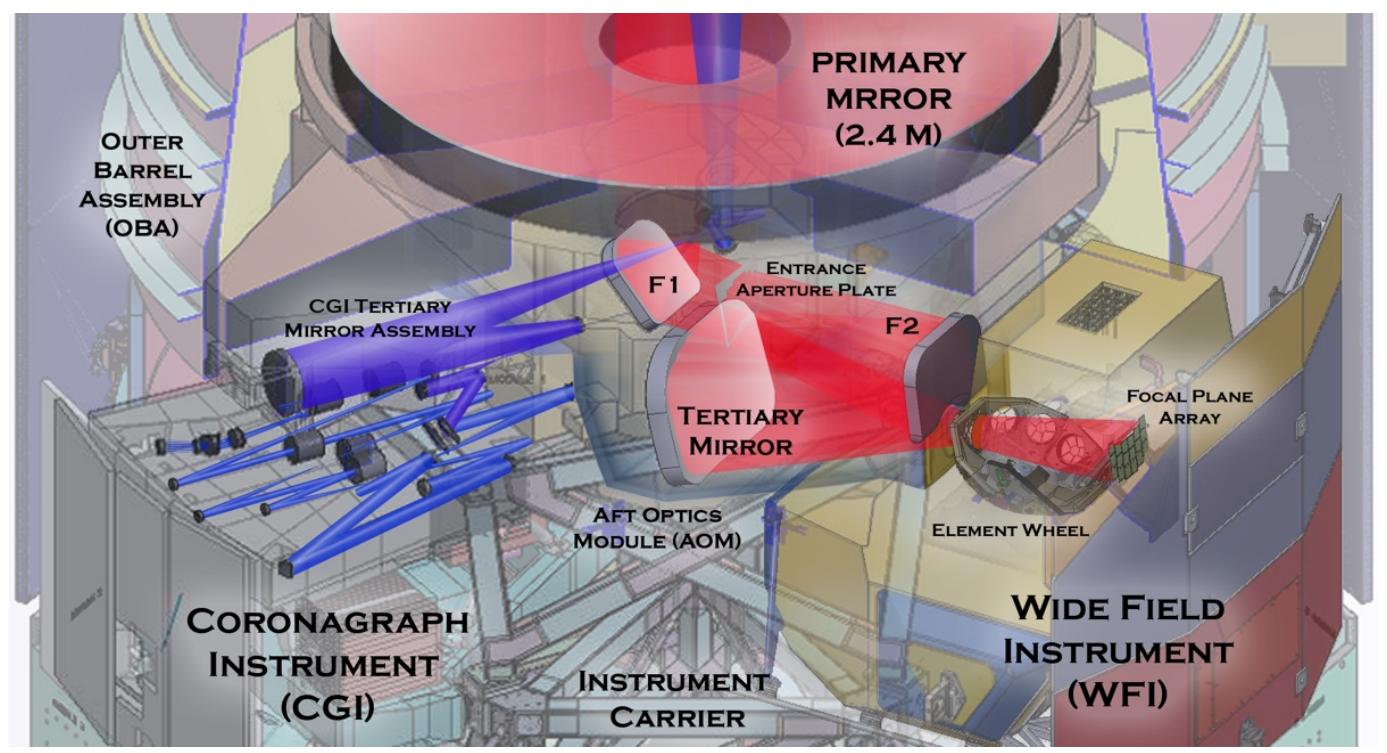

Figure 4. The WFIRST Phase-A Observatory showing the telescope, Wide Field Instrument (WFI) and Coronagraph

Instrument (CGI.) Note the Tertiary Mirror is integral to the IOA, with all WFC mirrors in a common meridian plane.

In the pre-Phase-A WFIRST architecture concept re-using the donated FOA, the Tertiary Mirror of the TMA was mounted within the WFI assembly for the Wide Field Channel. When the WFI was moved off of the OTA and into the IC, analysis revealed an increased sensitivity to observatory thermal and dynamic perturbations due to the metering of the WFI independently of the OTA. 2016 trades supported the decision to reduce risk by moving the WFC TM out of the WFI and into the OTA via the addition of the Aft Optic Module. A functional block diagram of the resulting WFIRST payload is illustrated in Figure 5.

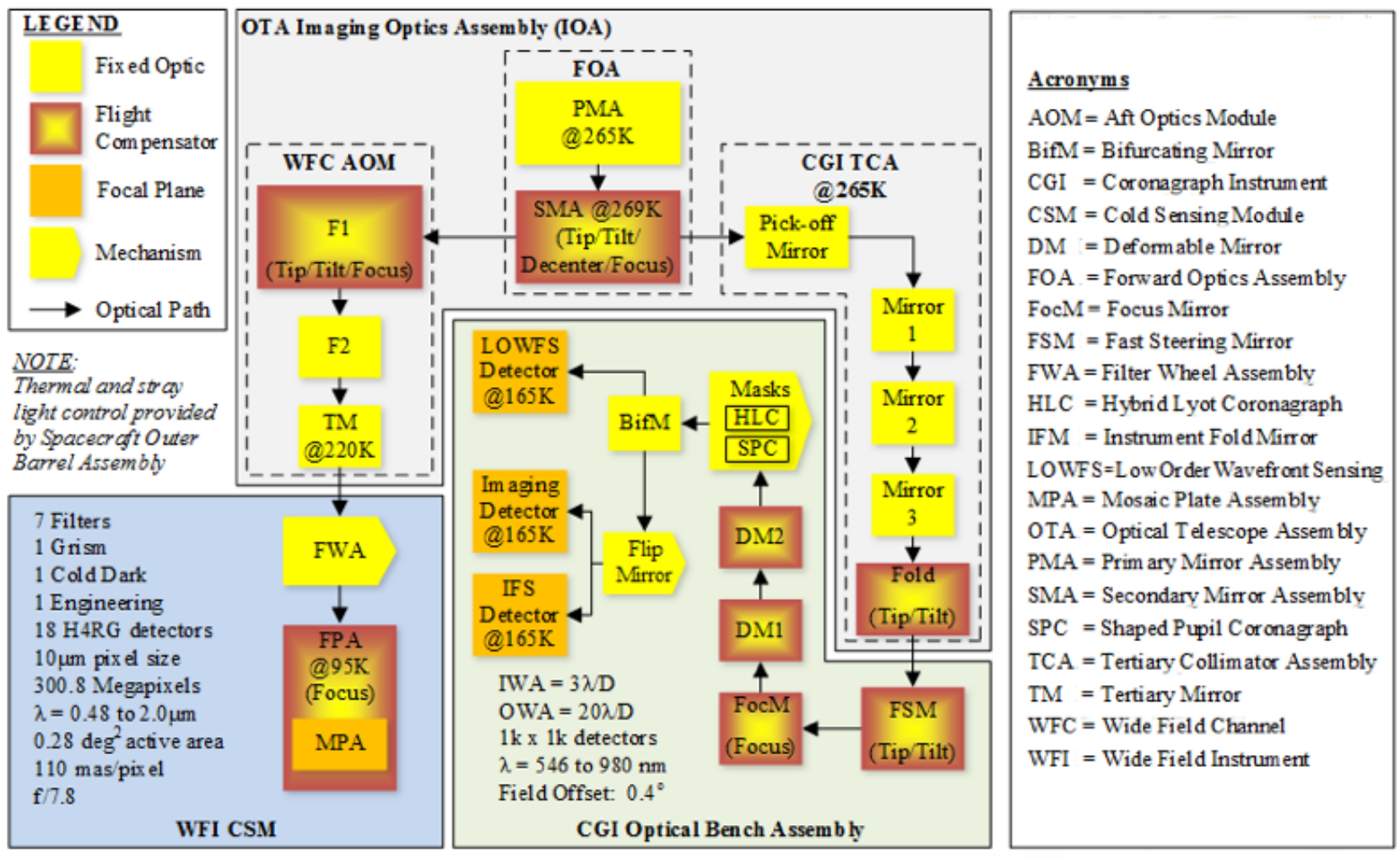

Figure 5: WFIRST Payload Functional Block Diagram. 
As shown in the block diagram, numerous on-orbit optical compensators are available to ensure the WFIRST Payload meets on-orbit alignment, optical quality, and long term optical stability requirements. The FOA secondary mirror provides a five degree-of-freedom rigid body adjustment capability and the AOM includes 3-DOF actuation on Fold Mirror 1. In the WFI channel the FPA provides alignment and focus adjustment, enabling on-orbit focus diversity phase retrieval during Observatory commissioning and as required thereafter.

The flight serviceable WFI consists of a Cold Sensing Module (CSM), a Warm Electronics Module (WEM) mounted within the spacecraft (not shown), and a Facility Cryogenic Radiator (FCR) mounted to the Spacecraft's Outer Barrel Assembly (not shown). All optical functions of the WFI are contained within the CSM.

The Filter Wheel Assembly (FWA) consists of at least 10 selectable modes; seven filters covering 0.48 to 2.0 microns, a grism for spectrographic studies, a cold dark for calibration, and an engineering filter used for ground AI\&T.

The Coronagraph Instrument (CGI) consists of the flight serviceable Optical Bench Assembly (CGI-OBA) and the Tertiary Collimator Assembly (TCA). The TCA optically and mechanically interfaces with the FOA to provide a collimated (infinite conjugate) input to the CGI. The TCA mounts directly to the FOA, and is hence not flight serviceable.

\section{CONFIGURATION MODIFICATIONS}

\subsection{Instrument Carrier}

Early in the pre-Phase A study it was quickly realized that the combined launch loads of the WFI and CGI would exceed the heritage design capability of the donated telescope. Various configurations of an Instrument Carrier (IC) to meter the WFIRST instruments off the spacecraft deck were subsequently traded. Our selected IC architecture (Figure 6(a)) leverages GSFC recent experience with the James Webb Space Telescope Integrated Science Instrument Module (ISIM) structure. The IC accommodates the IOA, WFI, CGI, and the Spacecraft's Star Tracker/Inertial Reference Unit (ST/IRU) via flight serviceable mounting interfaces (Figure 6(b)).
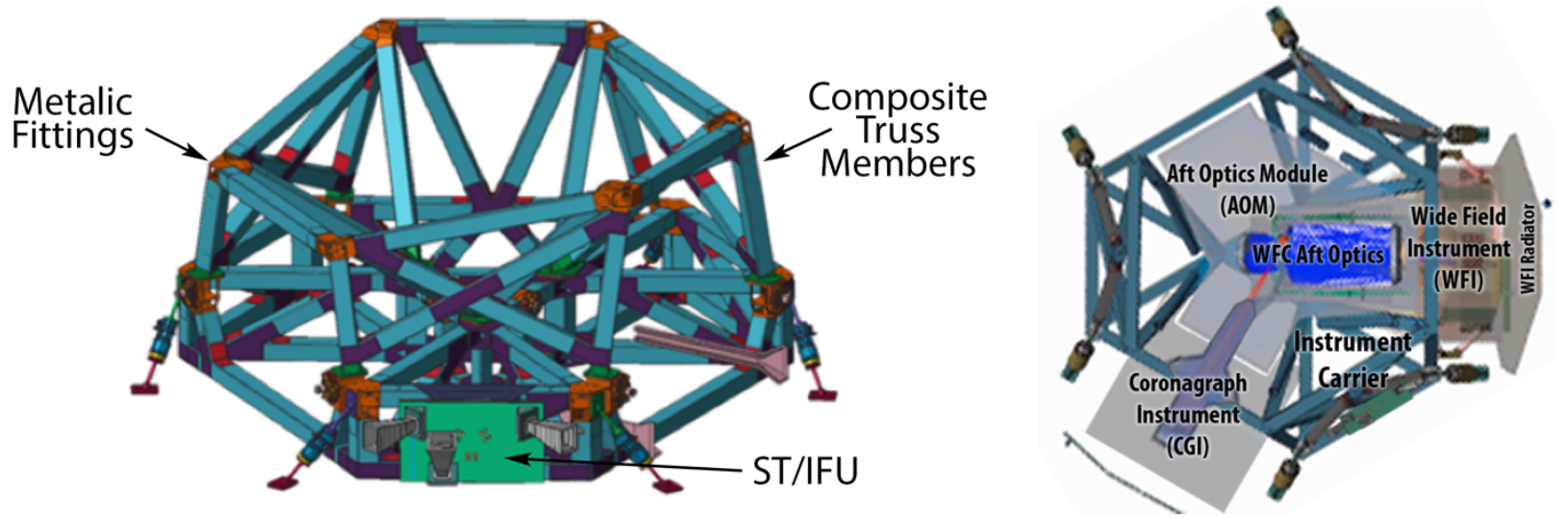

Figure 6(a) The WFIRST Instrument Carrier (IC). (b) IC Top View showing the AOM, WFI and TCA/CGI.

The IC is kinematically mounted to the WFIRST Spacecraft and consists of a carbon fiber/cyanate ester composite (M55J/954-6) truss assembly to achieve high levels of structural strength and stability in a lightweight structure. This is the same material used for the JWST ISIM, with the same lay-up but with some of the composite tubes having a larger OD. Truss members are joined with a combination of titanium fittings and composite gussets and clips; again with design heritage in the JWST/ISIM; however WFIRST will use titanium fittings in lieu of invar to conserve mass. Analysis has shown that titanium works at expected IC temperatures throughout the WFIRST mission, and our analysis will be augmented by test articles currently in fabrication. Dynamic isolation of the IC from the Spacecraft is achieved using Honeywell D-struts.

\subsection{Wide Field Instrument Optical Interface}

The Pre-Phase-A WFC design had the disadvantage of being a "divided" telescope; the tertiary mirror was mounted within the WFI assembly, apart from the primary and secondary structure. Because the instrument interface was at the 
caustic intermediate image, a series of steps involving holographic optical elements and translation stages were outlined to verify the requirements for the both Wide Field Instrument and the telescope. While these I\&T and wavefront sensing methods are in themselves robust, they accounted for several additional terms to the error budget, also affecting schedule and cost.

Key outcomes of the move of the WFC TM from the WFI to the OTA included reduced OTA to WFI alignment tolerances, simplification of Payload AI\&T by establishing a test point in the AI\&T flow that allowed double pass interferometry of the entire image formation system ("test-as-you-fly" (except for gravity release)), and the elimination of costly support equipment intended to compensate for the absence of the TM during Payload AI\&T. However, the most important outcome of the WFC TM move was not initially anticipated. The packaging of the OTA and WFI now allowed for the positioning of the WFI Mosaic Plate Assembly (AKA the WFI's 95K focal plane) much farther outboard towards the anti-sun side of the Observatory. It was quickly realized that this could enable the largest risk reduction achieved for WFIRST since 2013, the elimination of the Observatory's cryo-cooler in favor of passive cooling of the WFI cold electronics.

\subsection{Passive Cooling Trade}

The use of passive cryo-cooling to operate the WFC and IFC SCA s at $\leq 100 \mathrm{~K}$ was first seriously considered when the WFIRST mission orbit was changed from GEO to L2 in 2015. That change was based primarily on considerations related to SCA radiation tolerance, but it also afforded the mission with a more stable thermal environment. In particular, it offered the possibility of a true cold side of the observatory that would only be exposed to deep space.

Mechanical Cryo-cooling (MC) using a NICMOS-heritage Turbo-Brayton system was the baseline at the time, primarily due to the extremely low vibration of its non-reciprocating design, the ability of its heat transfer lines to provide cooling to focal planes located deep within the Payload, the tolerance of the high temperature (nearly room temperature) MC radiator to GEO thermal loading variations, and the relatively high technological maturity of the planned implementation.

With the move to L2, a trade was conducted to evaluate the MC concept against Passive Cryo-cooling Systems (PCS) employing either cryo heat pipes or traditional thermal straps. The recommendation at the time was to retain the MC concept, as the cryo heat pipes were a less mature technology, and the temperature gradient in the thermal straps given the inboard location of the SCAs was too large to allow an acceptable cryo radiator size. However, it was specifically noted in the trade closure that a PCS option could be reconsidered if the SCAs could be located closer to a PCS radiator, and the telescope outer barrel assembly could be made to support a large $\left(\sim 9 \mathrm{~m}^{2}\right)$ PCS radiator, and/or the SCA operational temperature could be increased to $\sim 120 \mathrm{~K}$.

The optical redesign activity to relocate the WFC TM offered the possibility of developing and evaluating configurations that would also move the SCAs radially outboard, much closer to the potential location of a PCS radiator (dubbed the Facility Cryogenic Radiator, or FCR, which would remain as part of the observatory if/when the WFI were changed out during servicing). When this SCA relocation proved practical, the mechanical vs. passive cryo-cooling trade was reopened and resulted in a change from MC to PCS SCA cooling. The primary advantages of the PCS concept were the unlimited life and zero intrinsic vibration, and the lower cost and complexity relative to MC.

It was realized that the PCS concept needed time to mature, so the MC and PCS options were carried in parallel for $\sim 9$ months in case unanticipated PCS issues were discovered. The new optical design was specifically chosen to be compatible with either a MC or PCS approach, enabling a change back to MC later in Phase A, should it be needed. Fortunately, the PCS design has remained robust, with parasitics margins fluctuating in the $110 \%$ to $130 \%$ range despite a lowering of the SCAs operational temperature from $100 \mathrm{~K}$ to $95 \mathrm{~K}$ (to improve SCA production yield), and a reduction in the size of the FCR from its original $9.5 \mathrm{~m}^{2}$ to its current $7 \mathrm{~m}^{2}$.

\subsection{WFC Fold Mirror 1 Assembly Actuation Mechanism}

Alignment trade studies during Phase- $\mathrm{A}^{[8]}$ also indicated the advantage of including 3-DOF actuation on the WFC F1. This is the baseline for the Phase-B as has been shown in the Figure 5 block diagram. Focus/tip/tilt of F1 allows for the $\mathrm{TM}$ to be aligned with respect to the PM/SM in the FOA. 


\subsection{WFC Focal Plane Assembly Actuation Mechanism}

The previous WFC design used an 3-DOF Instrument Focus Mirror prior to the fixed-position FPA. With the selection of Ball Aerospace as the WFI vendor, this has been eliminated from the optical path entirely in favor of a direct 6-DOF actuated FPA assembly. Using JWST-heritage hexapod hardware, the entire FPA will be aligned to the IOA output image. This benefits the alignment budget for commissioning and on-orbit stability.

\subsection{Integral Field Channel Loss}

To maintain project scope within the cost constraints, the Integral Field Channel (IFC) was removed from the Payload. The optical system was previously described ${ }^{[6]}$. Trades are now underway with the optical design and science teams to determine the most beneficial method to do adequate non-IFC Supernova type-1a redshift spectroscopy, including from the ground and/or using a low-dispersion element in the Wide Field Channel.

\section{PHASE-B WIDE FIELD CHANNEL (WFC) DESIGN AND PERFORMANCE}

Phase-A brought major changes to the observatory architecture. As we anticipated the roadmap to the Phase-B protoflight design work, a Payload Opto-Mechanical Working Group evaluated the effects of any inter-disciplinary analyses that would drive adjustments to the optical design, though such adjustments were minor and did not change the overall architecture. These included mechanical clearances, instrument interfaces, wavefront sensing and stray light, as well as thermal, dynamic and integrated modeling results. Acquisition strategy decision for various components and subsystems were considered, and simulations from the science community also provided feedback to our engineering trades.

\subsection{Optical Layout}

The AOM houses the WFC Aft Optics. The first fold mirror (F1) acts as a pickoff for an off-axis section of the field, where the Entrance Aperture Plate (EAP) acts as a field limiter and stray light baffle for the WFC at the intermediate "Cassegrain" focus. A second fold mirror (F2) folds the bundle back towards the axis, where the only powered mirror within the AOM, the Tertiary Mirror TM (R: $1643.2784 \mathrm{~mm}, \mathrm{~K}:-0.5965290831)$ works in concert with the PM \& SM to produce the corrected WFC image. All three powered mirrors of the TMA are simple conics.

By minimizing the distance from F2 to the TM, the TMA exit pupil is able to be projected outside of the AOM. Once the ray bundle passes the telescope exit pupil, it enters into the Wide Field Instrument. The WFI optics consists of a mechanized Element Wheel, and Focal Plane Array (FPA) with a mechanism to provide focus adjustment on-orbit.

\subsection{Widefield Imager Mode (WIM) Nominal Performance}

By offsetting the SCA columns, the Field of View layout follows the natural annular curve of an off-axis TMA optimized field. Small non-axial adjustments are used to further optimize the active area of the field. We maintained a $95 \%$ (RMS) polychromatic wavefront error of $<45 \mathrm{~nm}$, half of the $90 \mathrm{~nm}$ budget (based on $1 / 13^{\text {th }}$ wave at $1.2 \mu \mathrm{m}$ ), across the entire field. The optical design residual imaging performance of the Wide Field is shown in Figures 7 \& 8:

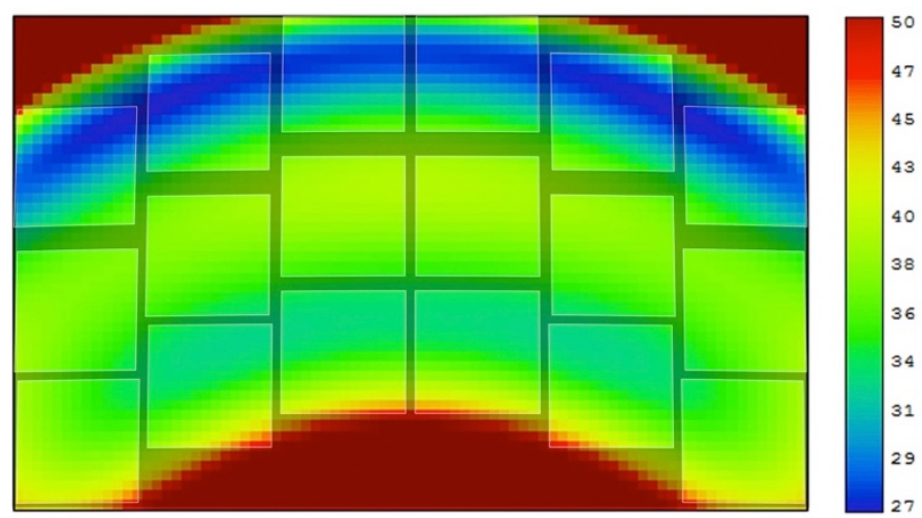

Figure 7. Optical design nominal performance of the Wide-Field Imager in RMS WFE (nm) The projected FOV of each sensor (1-18) is overlaid, also showing the effects of $\sim 2 \%$ field distortion. 

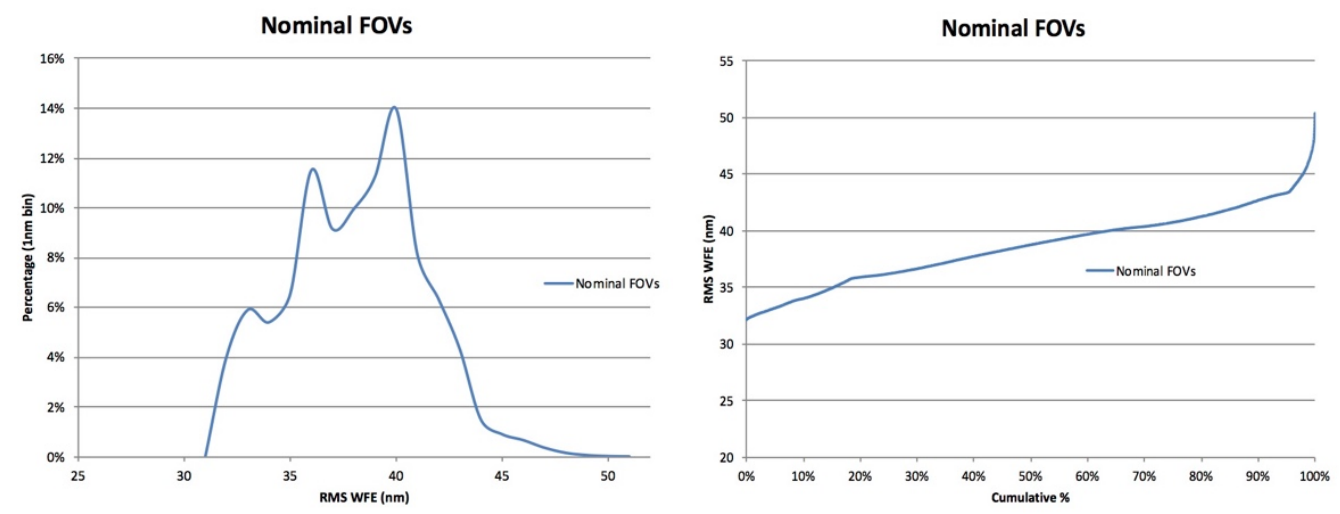

Figure 8. (a) Histogram of RMS over the field, and (b) Cumulative RMS performance as a percentage of field coverage.

The grism has been redesigned to optimize various fabrication and I\&T parameters. The performance requirements are looser than the WIM $(<150 \mathrm{~nm}$ RMS), however performance over much of the field significantly exceeds the requirement. (See Figure 9.)
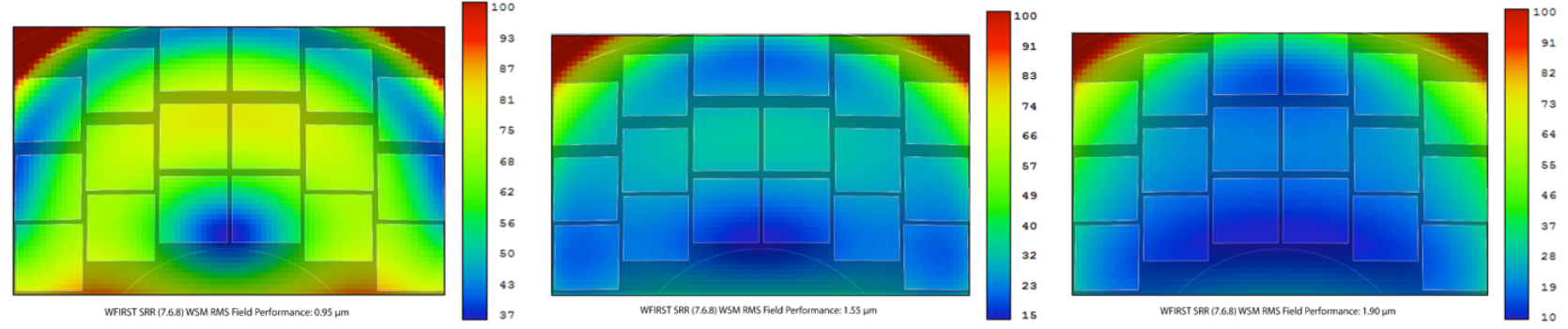

Figure 9. Optical Design Residual Performance of the WSM in RMS WFE (nm) at short, mid, and long wavelengths.

\section{OPTICAL MODELING ALLOCATIONS}

The modeling of the optical system is ultimately concerned with the final figure and orientation of each optical element relative to the motion of the structure. We first use a sensitivity table to make allotments for ranges, accuracies and resolutions. The Monte Carlo optical model then allows independent optical element perturbations and compensation in any desired coordinate system. This is also true of grouping of optics based on the grouping of the AOM and WFI optical bench in relation to the telescope Aft Metering Structure (AMS) in connection through the instrument carrier. The EW and additional opto-mechanical grouping and breakdowns can be added to the model as desired.

We have broken the process of performance allocation into three "Phases:"

Phase 1 - Fabrication \& Alignment

Phase 2 - Pre-Launch: Models, Testing and Pre-Launch Bias

Phase 3 - Post-Launch: Commissioning and Operations

\subsection{Phase 1: Fabrication \& Alignment Allotments}

First we consider fabrication allotments for each optical element. The ranges are based on state-of-the-art fabrication techniques and metrology. Measurements performed internally at GSFC (such as Cryogenic High Accuracy Refraction Measuring System (CHARMS) refractive index measurements. ${ }^{[9,10]}$ ) and vendor certifications are considered. The applied ranges and known measurement precisions are compensated by other parameters as per the compensation matrix (based on current I\&T plans.) Measurement Accuracies (RSS values) and Resolution (the smallest resolvable measurement) are generally considered residual errors and applied uncompensated, except for focus removal (because of the active mechanism on the FPA.) 
Likewise, the Alignment ranges, precisions and accuracies are accounted for via a compensation matrix consummate with the envisioned alignment plan. Values are based on state of the art alignment metrology including spatial analysis software developed at GSFC. These allocations cover the I\&T bookkeeping for all alignment capabilities, as any phase will re-check alignment to the same precisions and accuracies.

\subsection{Phase 2: FEM Modeling, Testing and Pre-Launch Biasing Allotments}

During this development time (prior to an engineering unit build), cooldown and gravity release perturbations are fully based on Integrated Modeling via Finite Element Method (FEM) model analysis (including cooldown, gravity release and outgassing). The FEM node points for each optical element are applied for both the rigid body motion and for surface figure deformation. MUF (Margin of Uncertainty Factor) values are applied as a multiplication factor to the FEM values for a "worst-case" perturbation range. While they may start out very high (e.g., 2.0), they are reduced by testing and model verification. Following testing (including thermal testing and gravity flip model verifications) and model refinements (including material properties and increased detail), the MUF values are expected to settle in the 1.15 -1.4 range (based on experience with JWST).

FEM Contributions are given compensated ranges to simulate the main I\&T and wavefront sensing (WFS)-based procedures:

1) IOA initial alignment, using full-aperture Auto-collimating Flat.

2) WFI: Pupil and FPA alignment test via induced stimulus.

3) Payload Full Double-Pass Test Simulation: Compensation by Telescope Focus (via SM), and WFC Focus (via FPA) and pre-launch alignment of WFI Bench (if necessary). Note that SM is a common element to all instrument channels, and so is used only to correct for both channels.

At this point, it is assumed that wavefront sensing will verify the alignments to a given residual value. ${ }^{[8]}$ To prevent compensation moving beyond the range of the actuators, various components may be biased with shimming to achieve system performance once on orbit. It is highly undesirable in the actual I\&T procedure to "re-do" shims following a verification test, so performance allocations are based on the "worst-case" of having to use only mechanized compensators.

\subsection{Phase 3: Commissioning and Residual Errors Allotments}

In the "post-launch" phase, we provide allotments for unknown errors in modeling, adding a "Residual MUF" - small amounts of perturbations to simulate the predicted vs actual final placements due to launch, cooldown, gravity, outgassing, etc. Compensation is based on wavefront optimization by mechanisms only (SM / F1 / FPA).

Once commissioning is final and observations have begun, any further perturbations are applied as uncompensated sensitivities. It is undesirable to perform "housekeeping" re-adjustment of any optics until a complete annual viewing cycle is complete, if even then. These include the periodical thermal fluctuations, caused by varying halo orbit positions and viewing angle slews. These also include long-term drifts of post-commissioning residual outgassing (based on FEM simulation) and any other unknown static or fluctuating changes. The exception may be the SM focus due to the SM support tubes' dL/dT. Structural jitter is carried as an a separate item in the system budget (see next section).

The areas of analysis are summarized in Table 1:

Table 1. Distinctions in Performance Allocation Modeling in Each Phase.

\begin{tabular}{|c|c|c|c|}
\hline & $\begin{array}{c}\text { PHASE 1 - Fabrication \& } \\
\text { Alignment Capabilities }\end{array}$ & $\begin{array}{c}\text { PHASE 2 - Pre-Launch: Models, } \\
\text { Testing and Pre-Launch Bias }\end{array}$ & $\begin{array}{c}\text { PHASE 3 - Post-Launch: } \\
\text { Commissioning Activities }\end{array}$ \\
\hline Perturbations & Based on I\&T and Optical Metrology & Based on FEM Models and WFS & Based on FEM Models and MUFs \\
\hline Compensators & $\begin{array}{c}\text { All Compensators are available, } \\
\text { Including Pre-figuring }\end{array}$ & $\begin{array}{c}\text { Select Compensators are available to } \\
\text { Pre-bias for predictions }\end{array}$ & $\begin{array}{c}\text { Only Mechanisms are Available for } \\
\text { Compensators - Tel: SM / F1 WIM: FPA }\end{array}$ \\
\hline Uncompensated & Focus is always removed & Focus is always removed & Focus is not removed for stabilities \\
\hline
\end{tabular}




\section{INTEGRATED MODELING RESULTS}

Integrated Modeling (IM) is analysis that includes multiple disciplines and uses a flight observatory model to provide end-to-end perturbation to performance predictions. The WFIRST Project has developed the IM processes and capabilities in the last several years to validate requirements that cannot be verified by test on the ground. The tool is also used to support system-level trades by performing observatory performance evaluation for different proposed designs. During Phase A of the mission, the IM analyses focused on the thermal elastic effects due to ground-to-orbit and on-orbit temperature changes which distort structures and lead to optical alignment and surface figure errors, and the mechanical vibration effect that is generated from the spacecraft reaction wheels and propagated through the observatory structure which also degrades the optical performance.

For thermal distortion mitigations, WFIRST employs a thermal control system with proportional heaters on the optical telescope assembly. For reducing jitter disturbances, WFIRST implements a two-stage, passive vibration isolation system, where the first stage is co-located with the reaction wheels, and the second stage is between the spacecraft and payload interface. In order to maintain stable performance, WFIRST plans to avoid moving any mechanisms (e.g. spacecraft high gain antenna actuators and WFI filter wheel) during science exposures. All other errors due to long-term material changes or dry-out effects can be compensated by flight alignment actuators as necessary.

The key stability requirements for the WFIRST Wide Field Instrument and Coronagraph Instrument (CGI) are summarized in Table 2. The CGI has internal control systems that can correct for line-of-sight (LOS) and wavefront (WFE) drift, outside of the observatory stability mitigation capabilities. In Phase A, to simplify the modeling approach, the CGI control systems are modeled as simple high-pass rejection filters. Both CGI requirements and performance predictions include these closed-loop rejection filters where appropriate, as shown in Table 2.

Table 2. WFIRST Key Stability Requirements

\begin{tabular}{|l|c|c|}
\hline \multicolumn{2}{|c|}{ Wide Field Instrument (WFI) } \\
\hline Title & Performance Requirement & Current Best Estimate \\
\hline $\begin{array}{l}\text { Wide Field Imaging Mode (WIM) Wavefront } \\
\text { Error (WFE) Stability (< 180 sec) }\end{array}$ & $1.0 \mathrm{~nm}$ RMS & $0.7 \mathrm{~nm}$ RMS \\
\hline WIM Long Term WFE Stability ( $>24$ hours) & $26.5 \mathrm{~nm}$ RMS & $7.2 \mathrm{~nm}$ RMS \\
\hline WIM Line-of-Sight (LOS) Jitter & $12 \mathrm{mas}$ RMS & 5.5 mas RMS \\
\hline WIM WFE Jitter & $0.9 \mathrm{~nm}$ RMS & $0.7 \mathrm{~nm}$ RMS \\
\hline \multicolumn{1}{|c|}{ Coronagraph Instrument (CGI) } \\
\hline Title & Performance Requirement & Current Best Estimate \\
\hline CGI WFE Drift (includes closed-loop filter) & Z4: 2.0 nm RMS & $\begin{array}{c}0.45 \mathrm{~nm} \text { RMS } \\
0.05 \mathrm{~nm} \text { RMS }\end{array}$ \\
\hline CGI LOS Jitter (includes closed-loop filter) & $\begin{array}{c}0.57 \text { mas RMS } \\
\text { RSS(Z5-Z11): 0.25 nm RMS }\end{array}$ & $\begin{array}{c}0.42 \mathrm{mas} \mathrm{RMS} \\
\text { (wheel speed: 5-19 rev/sec) }\end{array}$ \\
\hline CGI WFE Jitter & $0.25 \mathrm{~nm}$ RMS & $\begin{array}{c}0.07 \mathrm{~nm} \text { RMS } \\
\text { (wheel speed: 5-19 rev/sec) }\end{array}$ \\
\hline
\end{tabular}

As part of the IM process, all prediction results include appropriate model uncertainty factors (MUFs). The MUFs are determined from heritage data and relevant past experience at this stage of the program. More extensive analyses will be performed during Phase B of the mission to ensure that the MUFs chosen are sufficient for WFIRST. With the MUFs included, the IM current best estimates as shown in Table 2 demonstrate that all key stability requirements can be met with reasonable margin. To meet the CGI LOS and WFE jitter requirements, the observatory wheel speed range is limited to 5-19 rev/sec, from the nominal $+/-40 \mathrm{rev} / \mathrm{sec}$. This wheel speed range is predicted to allow CGI to achieve its technology demonstration goals. 


\section{STRAY LIGHT}

The Phase-B Stray Light modeling has played an important role. First, it has allowed the baffling of the FOA exclusion fields to be relaxed, minimizing the size of the central obscuration. The study of thermal emissions per waveband effected the decision to maintain independent masking on each filter position. More discussion on this and other results will be presented in future papers.

\section{SUMMARY AND CURRENT EFFORTS}

In summary, great progress has been made by the entire WFIRST team of scientists, engineers, and our empowering staff of managers to progress the design of the WFIRST observatory while reducing technical and programmatic risk, meet all science objectives, and satisfy our stakeholder commitments on mission cost and schedule. Since 2013 notable advances in observatory design, packaging, and performance has been achieved as reported herein, and the WFIRST Mission is now poised for an exciting and productive Phase B, leading to Mission PDR in late 2019. In Phase-B, we are focusing our efforts on the documentation of the optical sensitivities and predictive performance modeling.

\section{ACKNOWLEDGEMENTS}

We sincerely acknowledge that this study is the product of a well-coordinated and growing team. This includes the WFIRST Formulation Science Working Group, team co-members of many disciplines, contractors, and project leadership and support staff. A special thanks goes to co-author Tom Casey for the majority of the systems discussion, to Dr. Dave Content for his guidance and advice in the preparation of this paper, Dr. Art Whipple for his help in editing, Mr. Clif Jackson for his help in ensuring the thermal trades discussed herein were captured accurately, and Dr. Alice Liu for her tireless attention to detail in providing a comprehensive summary of the WFIRST integrated modeling results for publication herein. The efforts of the optical/opto-mechanical engineering team of Nerses Armani, Guangjun Gao, John Hagopian, Alden Jurling, Cathy Marx, and Jacob Murry (summer 2018) have been essential and invaluable.

Other WFIRST payload team members include: W. Alexander, L. Bartusek, C. Blaurock, A. Carra, D. Content, C. Cottingham, J. Davis, M. Dipirro, S. Godo, J. Gygax, J. Hawk, G. Hobbs, C. Jackson, A. Jurling, J. Kruk, X. Li, M. Marzouk, C. McDonald, K. Mehalick, M. Melton, H. Peabody, C. Powell, S. Rohrbach, G. Rosanova, J. Ruffa, C. Tolman, M. Vess, (JPL:) Y. Conturie, R. Egerman, F. Forkl, T. Hahm, J. Mondello, S. North, S. Sutton, T. Wilson, P. Voyer, (Harris Corp:) R. Demers, R. Effinger, B. Kern, C. Noecker, I. Poberezhskiy, H. Tang, D. Braun. This work was funded by the National Aeronautics and Space Administration (NASA.)

\section{REFERENCES}

[1] Please refer to the WFIRST Project Page, $<$ http://wfirst.gsfc.nasa.gov $>$

[2] Content, D., Aaron, K., et al., "Wide Field Infra-Red Survey Telescope (WFIRST) 2.4-meter Mission Study," Proc. SPIE 8860, (2013)

[3] Content, D., Armani, N., et al., "Wide field instrument preliminary design for the Wide Field InfraRed Survey Telescope," Proc. SPIE 8860, (2013)

[4] Pasquale, B., Content, D., et al., "Optical Design of the WFIRST-AFTA Wide-Field Instrument," Proc. SPIE 9293, (2014)

[5] Pasquale, B., Marx, C., Gao, G., Armani, N., Casey, T., "Optical design of the WFIRST Phase-A Wide Field Instrument," Proc. SPIE 10590, (2017)

[6] Gao, G., Pasquale, B., Marx, C., Chambers, V., "Optical Design of the WFIRST Phase-A Integral Field Channel," Proc. SPIE 10590, (2017)

[7] Pasquale, B., Marx, C., Content, D., Kruk, J., "Widening our View of the Universe," Physics World Optics and Photonics Special Edition, June, 2016, <http://live.iop-pp01.agh.sleek.net/2016/05/19/widening-our-view-of-theuniverse $>$

[8] Hagopian, J., Armani, N., et al., "Ground to on-orbit alignment study of the WFIRST wide-field channel and resulting changes in the telescope architecture," Proc. SPIE. 10377, (2017)

[9] K. Miller, M. Quijada, D. Leviton, "Cryogenic refractive indices of S-LAH55, S-LAH55V, S-LAH59, S-LAM3, SNBM51, S-NPH2, S-PHM52, and S-TIH14 Glasses," Proc. SPIE 9578, (2015)

[10] Frank U. Grupp, et al., "The need for accurate cryo refractive indices and mechanical properties of optical materials (Lithosil 3001, $\mathrm{CaF}_{2}$ and S-FTM16 for EUCLID NISP)," Proc. SPIE, 9578, (2015) 